\title{
Acoustic emission signal source localization on plywood surface with cross-correlation method
}

\author{
Yang $\mathrm{Li}^{1}$ · Shuai-Shuai $\mathrm{Yu}^{1} \cdot \mathrm{Li} \mathrm{Dai}^{1} \cdot$ Ting-Fang Luo $^{1} \cdot \mathrm{Ming} \mathrm{Li}^{1}$
}

Received: 1 July 2017 / Accepted: 1 October 2017 / Published online: 5 January 2018

(c) The Japan Wood Research Society 2018

\begin{abstract}
The high-speed data acquisition equipment which is based on NI and LabVIEW software was used to establish the acoustic emission (AE) signal collection platform, and adopted wavelet analysis method to extract the weak of AE signal waveform from noise, aiming at the localization of AE source on plywood surface. The signal cross-correlation analysis method is used to determine the time difference between the AE signals and the different sensors,so that further calculates the average propagation speed of AE signal on the plywood surface. A kind of AE signal source localization method which is based on the cross-correlation method was proposed according to the principle of geometric positioning, and verified by means of the testing experiment which selected the lead break to simulate AE source. This paper has shown that wavelet analysis can effectively extract AE signal waveform, and the localization method based on cross-correlation principle can precisely calibrate the position of AE signal source.
\end{abstract}

Keywords Plywood surface $\cdot$ LabVIEW $\cdot$ Acoustic emission $\cdot$ Wavelet analysis $\cdot$ Cross-correlation location

\section{Introduction}

Acoustic emission (AE) is a phenomenon in which the transient elastic wave has been generated by the materials' localized source energy released rapidly [1]. Thanks to the dynamic nondestructive detecting method of AE technology, currently AE testing are widely used in the research of materials' internal defects, monitoring the strength of aircraft components and so on $[2,3]$. The main purpose of this testing technology is to determine the location of $\mathrm{AE}$ source, and also exert a crucial part in the evaluation of $\mathrm{AE}$ instruments, its accuracy directly reflects the degree of coincidence between the location of AE source and the actual position of the active defect source. It is a significant issue for $\mathrm{AE}$ detection and evaluation to improve the positioning accuracy of the $\mathrm{AE}$ source and minimize the leakage and the false location $[4,5]$. As a kind of natural renewable resources, wood has always been serving as a greatly important building material, especially the growing popularity of

Ming Li

swfu_lm@swfu.edu.cn

1 School of Machinery and Transportation Engineering, Southwest Forestry University, Kunming 650224, Yunnan, China wood structure is so quick that impels the safety monitoring to be more crucial and important. Nevertheless, in comparison with other solid materials, wood interior has anisotropy characteristic of elasticity and plasticity and the AE signal propagation speed has obvious discrepancy under the different environments [6-8]. Therefore, aiming at positioning the AE source of wood also faces a significant challenge.

For the most part, AE signal is divided into two types: burst and continuous. While often adopts to two kinds of location methods named as time-of-arrival location (TOA) and zone for the burst signal, utilizes the cross correlation principle to estimate the time difference both of AE signals so as to exactly locate the AE source of the continuous signal. As early as 1968, by arranging a set of probe arrays, quite a few scholars had determined the three-dimensional position of all kinds of $\mathrm{AE}$ events [9], additionally the research which explores the location methods of AE source has reached new heights in the past few years. Moreover, uses the method of short-time cross-correlation principle to reckon up the time difference, in which all sensors received the AE signal, then can quickly obtain the position of $\mathrm{AE}$ source in the range of error permissible [10]. Furthermore, other studies applied wavelet transformation theory and cross time-frequency spectrum (CTF) to orientate the $\mathrm{AE}$ source of plate-type structure varying wave velocity [11]. 
In the previous study, the optical fiber Bragg grating (FBG) sensor was used to establish the AE signal acquisition system of aluminum alloy, and established the equation of quadrilateral array and improved particle swarm optimization (PSO) algorithm, which achieved the localization and optimization of AE source [12]. Meanwhile, many domestic scholars have recently carried out relevant research. To name only a few, aiming at some characteristics which AE signal contained both multimodal and low signal-to-noise ratio (SNR), had proposed an AE source location method with which combined wavelet transform and threshold analysis [13]. Then, the whole phase-difference time delay estimation method was discussed, which effectively improved the accuracy of location of AE source [14]. On the other hand, the wood surface of AE localization method was put forward and verified on the basis of geometric positioning time [15]. A method based on time-reversal theory was proposed to accurately orientate the AE source on the detection of steel plate, as well as the position of it was revealed through the signal focusing [16].

Glulam has gradually become the main building material for gymnasiums and large public places, because of its uniform structure and high strength, the detection of its safety performance is essential. AE technology, as the only active nondestructive testing method, provides a feasible and effective way of security. However, most of the rock and metal are used as raw material to study the characteristics of AE signal and the localization of AE source at home and abroad, and research in the AE signal of wood is limited to solid wood, there are few reports on the localization of AE source for Glulam. Therefore, in the present study, plywood is selected as the experiment materials, and an exploratory research on the characteristics of $\mathrm{AE}$ signal and the localization of $\mathrm{AE}$ source is provided. We develop an acquisition platform of AE signal based on NI high-speed acquisition equipment in the first place, in addition, analyze the AE signal characteristics of the specimen, in which simulate AE source by breaking lead core, and apply the wavelet analysis to obtain the weak AE signal which submerges in noise. In the course of time, through the cross-correlation technology to receive the delay calculation between the AE signal of each sensor, so as to accurately locate the AE source.

\section{Materials and methods}

The test chose plywood as the research specimen, which was manufactured with dimensions of $500 \times 400 \times 15 \mathrm{~mm}^{3}$ (length $\times$ width $\times$ thickness). As the book recorded [17-19], the wood AE signal is mainly concentrated in the range of 50 to $210 \mathrm{kHz}$, in this connection, we use the AE sensor of single-ended resonant named by SR $150 \mathrm{~N}$, which the acquisition frequency is between 20 and $220 \mathrm{kHz}$. This sensor is

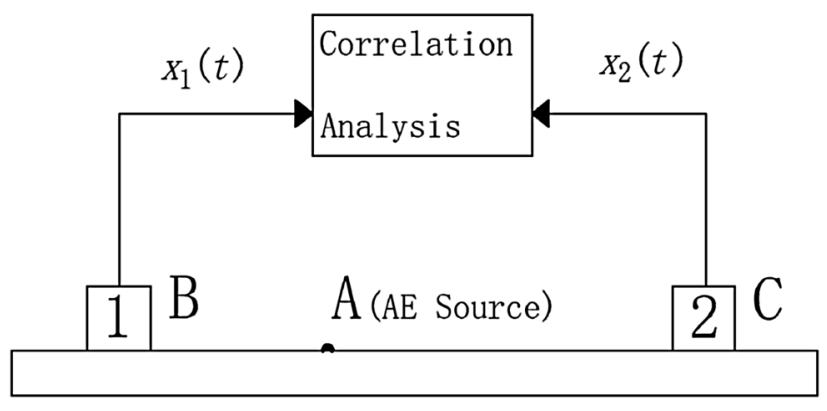

Fig. 1 Sensor placement and system connection diagram

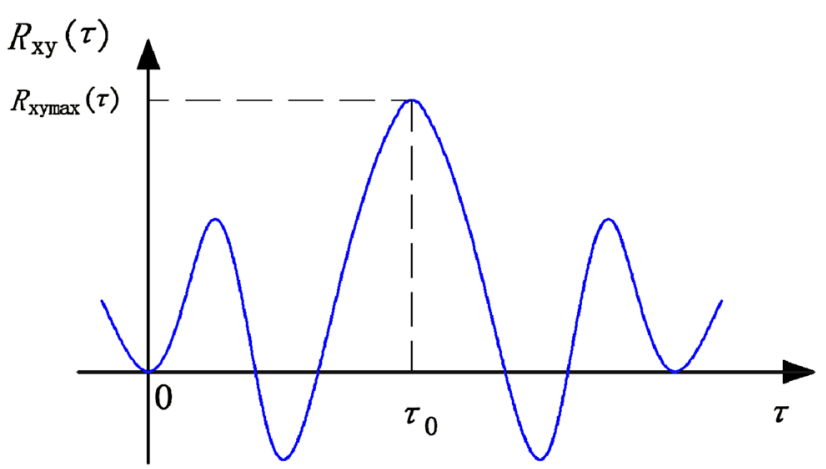

Fig. 2 Diagrammatic sketch of cross-correlation function

also equipped with a gain of $40 \mathrm{~dB}$ PAI preamplifier which has built-in noise reduction device, which not only can effectively amplify the signal, but also reduce the noise disturbance. Furthermore, the acquisition equipment of AE signal is USB-6366 NI high-speed device, the sampling frequency of which is up to $2 \mathrm{MHz}$. In the course of the entire process of the experiment, the AE source is simulated by the way of lead fracture, the acquisition voltage amplitude of each channel is the range of $\pm 5 \mathrm{~V}$, and the sampling frequency is approximately $500 \mathrm{kHz}$.

Based on the cross-correlation principle, the time differences or delay measurements can be carried out between the chopping and the continuous wave, which is expressed as the degree of correlation among the two time series, that is, described the degree of correlation between the random signals $x(t)$ and $y(t)$ at two different moments both $t_{1}$ and $t_{2}$ $[20,21]$. Thus, assuming that there is a defect at the point A, and the sensors 1 and 2 are installed at fixed points B and C, respectively, to accurately locate them (Fig. 1).

The distances of the location of the sensors 1 and 2 from point A are $S_{1}$ and $S_{2}$, respectively, under these circumstances (Fig. 2). Meanwhile, the response of the two sensors is $x_{1}(t)$ and $x_{2}(t)$, and the cross-correlation function diagram shows that the delay $\tau_{0}$ corresponding to the maximum value of $R_{x y}$ is the time difference between which AE source transmits from point $\mathrm{A}$ and two sensors, 
$S_{2}-S_{1}=v \times \tau_{0}$

( $v$ is referred to the propagation speed of the AE signal in the glued wood, which is known). In the experiment, the spacing $(S)$ of sensors can be directly measured by

$S=S_{1}+S_{2}$

and then both $S_{1}$ and $S_{2}$ can be solved, respectively. The accurate positioning of the sound source A can be realized. Thereby the position of point A called as AE source can be accurately located.

The sensor into a linear layout on the specimen surface in this article, and the simulation results are shown in Fig. 3. Soon afterwards the sensor is placed at the two points, $A$ and $\mathrm{B}$, and the AE source (point $\mathrm{O}$ is located at the midpoint of the two sensor lines) is simulated by the lead core breaking method in order of six points in $\mathrm{C}, \mathrm{D}, \mathrm{O}, \mathrm{E}, \mathrm{F}$ and $\mathrm{G}$. In order to reduce the measurement error, the middle point $\mathrm{O}$ which the coordinate origin is located by $(0,0)$, and the other points coordinates are selected in $(-15,0),(-5,0),(5,0)$, $(10,0)$ and $(17,0)$ respectively. Meanwhile, five experiments

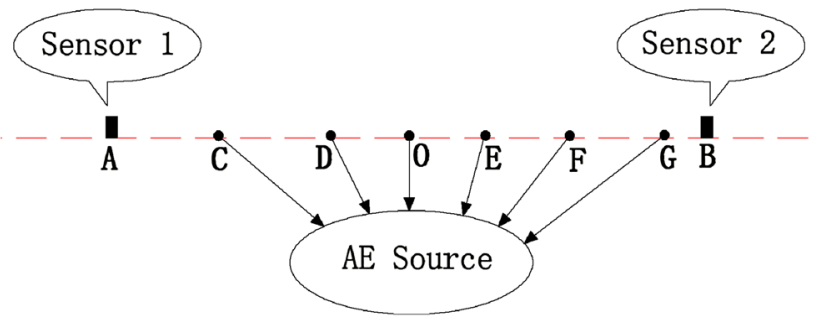

Fig. 3 Experimental scheme simulation diagram

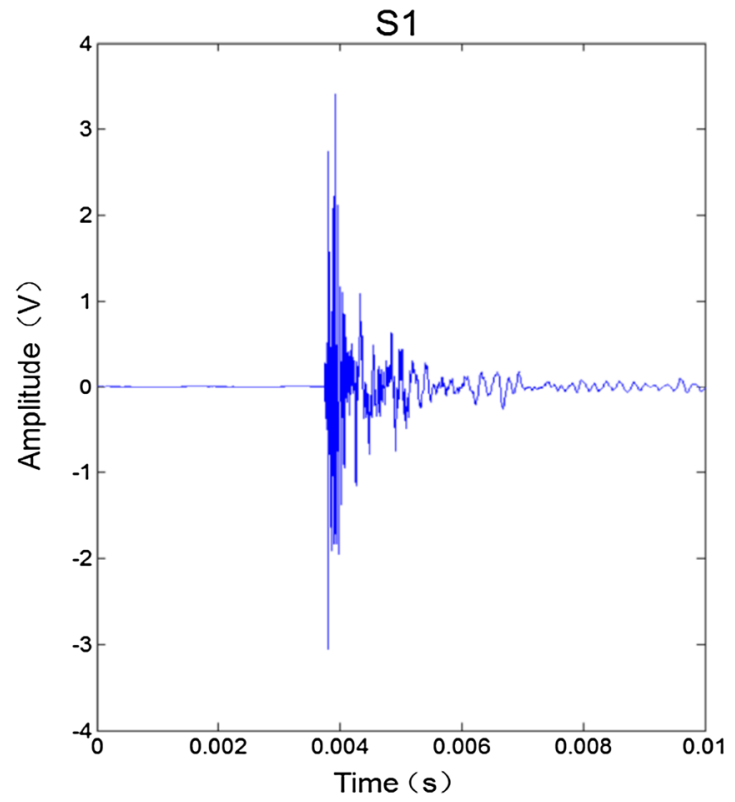

were carried out at each AE source, and a total of thirty sets of data were collected. Last but not least, designs the software platform of AE signal analysis and processes based on the LabVIEW software, and combines with the Matlab wavelet program to decompose them for the sake of further analyzing the time-frequency domain of the collected AE signal. This method which decomposes into signals of each frequency band and eliminates the signal band containing noise, reconstructs the signal frequency with AE signal, thus extracts the AE signal which is submerged in the noise.

\section{Results and discussion}

Affected by the complex structure inside the plywood, its AE signals are often "submerged" in noise due to severe attenuation. Figure 4 shows that the original AE signal acquired by the $\mathrm{AE}$ source at the point $\mathrm{C}$ within the broking lead test, and the AE signal of plywood is extremely weak and completely covers in noise. Accordingly, before the analysis of the AE signal, it needs to be pre-treated [22], and the signal should be extracted from noise to accurately calculate the propagation rate of the AE signal of the plywood to accurately achieve positioning effect. In this paper, db5 is chosen which is commonly used in Daubechies wavelet as the wavelet base to carry out 8-level wavelet decomposition, based on the absolute superiority of wavelet analysis in signal denoising. Moreover, the high-frequency coefficients are quantized with soft thresholds, and the AE signal is reconstructed by forcing the zero-removal channel coefficients and

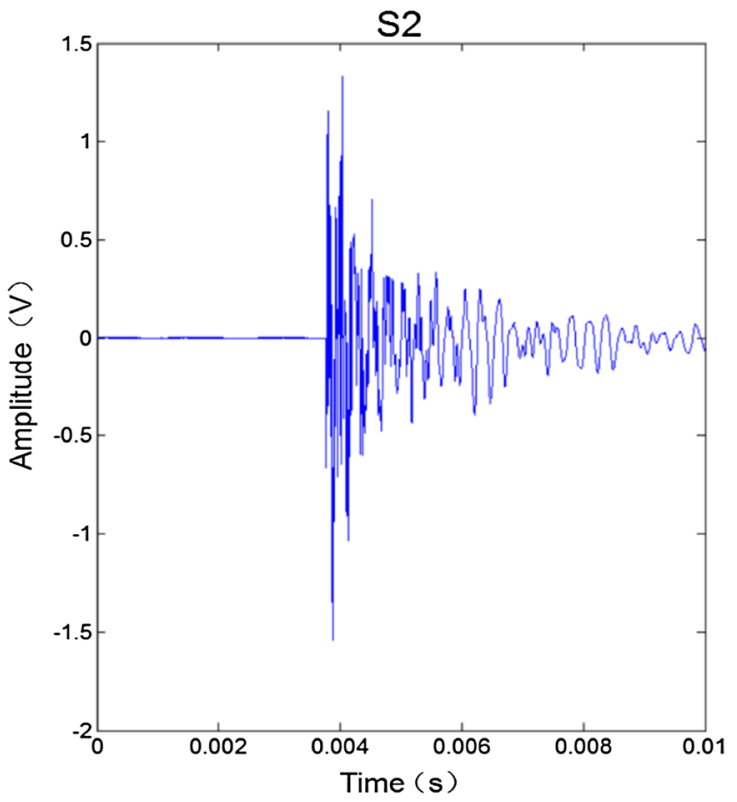

Fig. 4 Time domain of original acoustic emission signal 
the high-frequency coefficients which are processed with threshold quantization [23-25] (Fig. 5).

It can be analyzed from Figs. 4 and 5 that wavelet transform has an obvious effect on noise reduction of AE signal and gets rid of a large amount of noise and useless part, in addition, extracts it from noises. Furthermore, the most important thing is to maintain all of the original $\mathrm{AE}$ signal information, and the characteristics have not been destroyed. It can be learned from the spectrogram that the frequency which the AE signal of the plywood used in the experiment is mainly concentrated in the range of $50-150 \mathrm{kHz}$, maintains the basic characteristics of wood and provides the function for the noise reduction of wavelet transform; therefore, it is further verified that wavelet analysis is the best method of AE signal denoising [15, 26, 27].

In the article, the cross-correlation diagram of the first set of AE signal reconstructed through wavelet analysis is made, when the AE source is selected at point $\mathrm{C}$ (Fig. 6). From the abscissa corresponding to the maximum amplitude in the figure, the time difference which AE signal transmits to the two sensors is equal to $63.453 \times 10^{-6} \mathrm{~s}$. Similarly, the two-channel cross-correlation graphs from Group 2 to 5 data can be made, and the time difference between the two
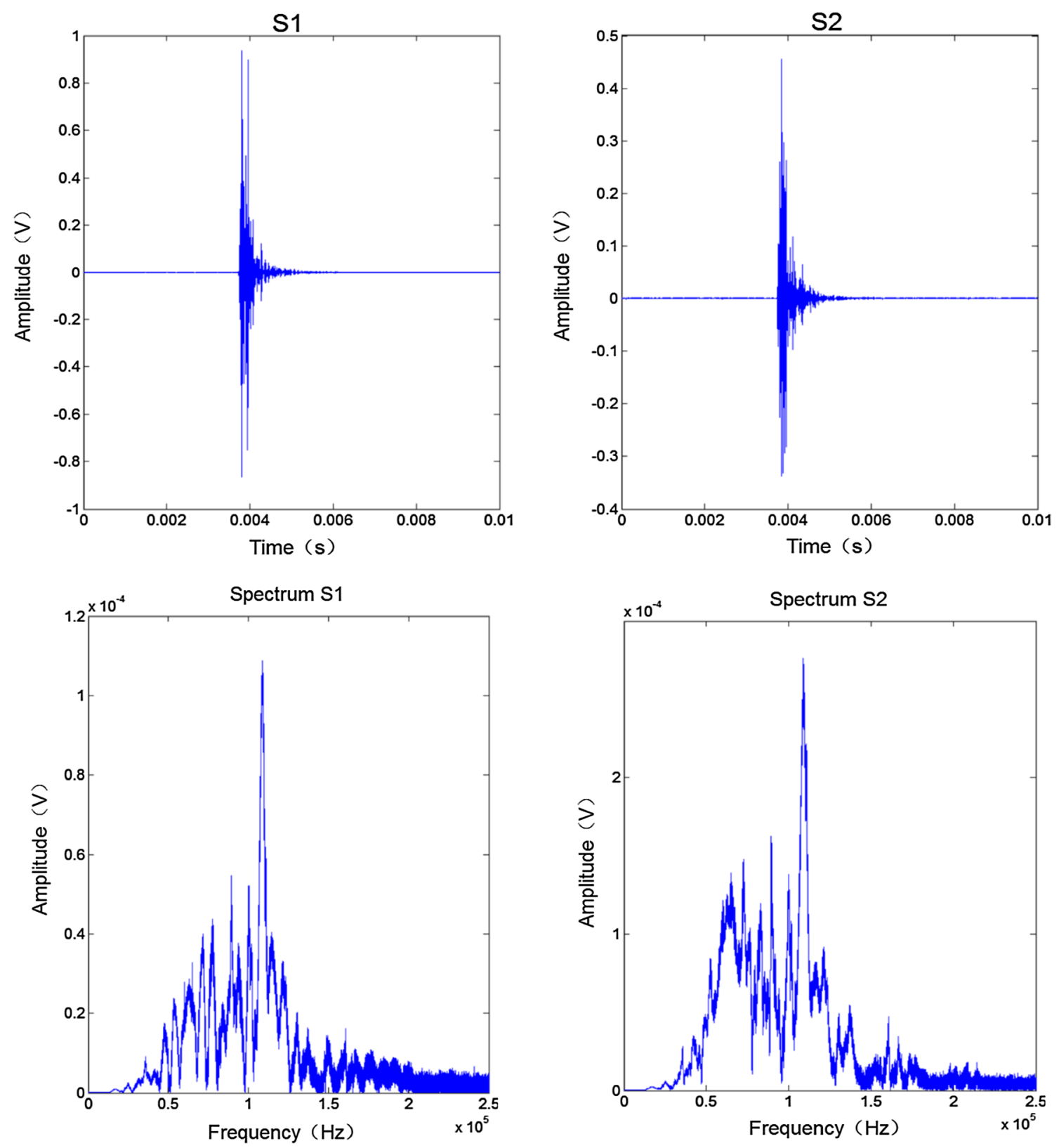

Fig. 5 Reconstruction time domain waveform and spectrum of AE signal after noise reduction 
Fig. 6 The cross-correlation diagram of point $C$ which the first set of AE signal after wavelet reconstruction

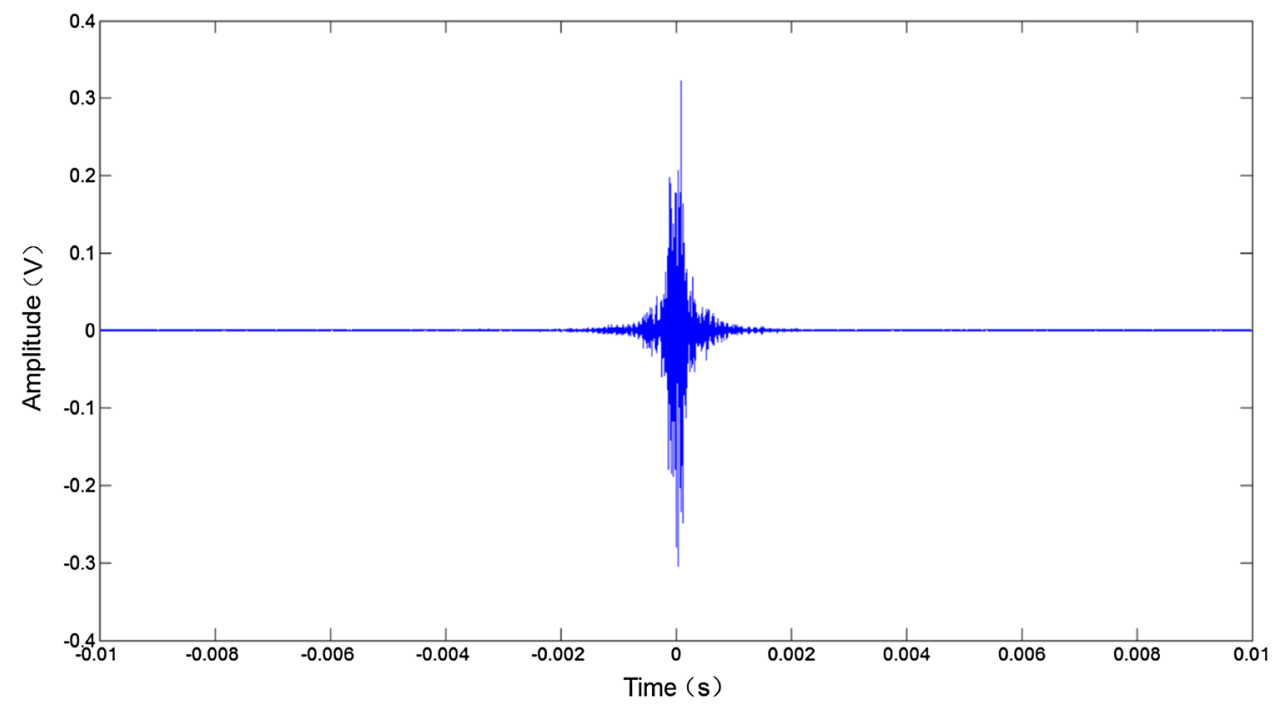

Table 1 Sound source for the point $\mathrm{C}$ of each group of experimental data for the two-sensor time difference

\begin{tabular}{ll}
\hline Experiment numbers & $\begin{array}{l}\text { The time difference of acous- } \\
\text { tic emission }(\mathrm{AE}) \text { signal }(\mu \mathrm{s})\end{array}$ \\
\hline 1 & 63.453 \\
2 & 62.643 \\
3 & 62.834 \\
4 & 63.525 \\
5 & 62.889 \\
\hline
\end{tabular}

sensors receiving the AE signal in each experiment can be precisely noted (Table 1 ).

Table 1 records the time difference at which the five groups of $\mathrm{AE}$ signals arrive at the sensor at point $\mathrm{B}$ of the $\mathrm{AE}$ source, thus the average time difference can be calculated as $63.069 \mu$ s. Due to the distance difference between the AE source of point $\mathrm{C}$ and the sensors $\mathrm{A}$ and $\mathrm{B}$ being equal to $30 \mathrm{~cm}$, the average velocity of the AE signal propagating in the plywood is $2357.326 \mathrm{~m} / \mathrm{s}$. And the accuracy of this result is compatible with the inability to accurately determine the position of each lead. The same can be calculated for the remaining five AE source simulation points of the AE signal time difference and the other data. Furthermore, as for the remaining five sets of experimental data analyses, the cross-correlation graph of the respective sensors receiving the AE signal is omitted and the calculated data are recorded directly in Table 2 , so as to prevent tautology.

Through the analysis of the above consequences, the use of cross-correlation technology on plywood AE source location has a significant effect. However, owing to the complex impacts of the internal structure of the plywood, the attenuation, scattering and other phenomena in the process of acoustic transmission, as well as some errors caused by man-made operation, such as the inability to accurately determine the position of each lead and the angle of lead, the positioning results show a random location without regular arrangement. The thirty sets of experimental data recorded in Table 2 show that three of the experimental data have large errors because of human operation or interference from the external environment, while $90 \%$ datum are within the range of allowable error (relative error is less than 4\%). Therefore, the accuracy of the cross-correlation technique for the AE source location of plywood is further verified.

\section{Conclusion}

The experiment applies cross-correlation technology only to locate the AE source on the plywood surface; it is possible to study the AE signal source location on the surface of fir, pine and other structural timbers, and to explore the characteristics of AE signals with different internal damage defects in the next step. The characteristic parameters of different types of AE signals among Glulam are established when combined with the results of this paper simultaneously, and provides reference for researching the internal damage and the location of the fracture areas at different materials.

This preliminary study introduces the common methods of signal source location for AE of wood surface, and the results show that the common method of time difference of arrival (TDOA) location is not suitable for the glued wood for which the type of AE signal is continuous. It can be seen from the spectrum of the AE signal of the plywood that there is large attenuation in the propagation process and that the spectrum is not a definite value, but rather a concentrated state, which mainly ranges from 50 to $150 \mathrm{kHz}$, to a greater extent limits the time difference method on the signal source positioning; hence, this paper proposes 
Table 2 The data processing results of sound source for point $\mathrm{D}$

\begin{tabular}{|c|c|c|c|c|c|}
\hline $\begin{array}{l}\text { Acoustic } \\
\text { emission (AE) } \\
\text { source }\end{array}$ & $\begin{array}{l}\text { Actual } \\
\text { coordinates } \\
\left(X_{\mathrm{i}}, 0\right)\end{array}$ & $\begin{array}{l}\text { The time differ- } \\
\text { ence of AE signal, } \\
\mu \text { s }\end{array}$ & $\begin{array}{l}\text { Coordinates after } \\
\text { calculating }\left(X_{\mathrm{i}}^{\prime}, 0\right)\end{array}$ & $\begin{array}{l}\text { Absolute } \\
\text { error }\left(X^{\prime \prime}{ }_{i} \text {, }\right. \\
0)\end{array}$ & Relative error, $\%$ \\
\hline \multirow[t]{5}{*}{$\mathrm{C}$} & -15 & 63.453 & -14.958 & 0.042 & 0.280 \\
\hline & -15 & 62.643 & -14.767 & 0.233 & 1.553 \\
\hline & -15 & 62.834 & -14.812 & 0.188 & 1.253 \\
\hline & -15 & 63.525 & -14.975 & 0.025 & 0.167 \\
\hline & -15 & 62.889 & -14.825 & 0.175 & 1.167 \\
\hline \multirow[t]{5}{*}{$\mathrm{D}$} & -5 & 21.155 & -4.987 & 0.013 & 0.260 \\
\hline & -5 & 21.261 & -5.012 & 0.012 & 0.240 \\
\hline & -5 & 22.072 & -5.203 & 0.203 & 4.060 \\
\hline & -5 & 21.580 & -5.087 & 0.087 & 1.740 \\
\hline & -5 & 21.732 & -5.123 & 0.123 & 2.460 \\
\hline \multirow[t]{5}{*}{$\mathrm{O}$} & 0 & 0.110 & 0.026 & 0.026 & - \\
\hline & 0 & 0 & 0 & 0 & \\
\hline & 0 & 0.687 & 0.162 & 0.162 & \\
\hline & 0 & 0.106 & 0.025 & 0.025 & \\
\hline & 0 & 0.369 & 0.087 & 0.087 & \\
\hline \multirow[t]{5}{*}{$\mathrm{E}$} & 5 & 21.609 & 5.094 & 0.094 & 1.880 \\
\hline & 5 & 35.069 & 8.267 & 3.267 & 65.340 \\
\hline & 5 & 21.011 & 4.953 & 0.047 & 0.940 \\
\hline & 5 & 21.261 & 5.012 & 0.012 & 0.240 \\
\hline & 5 & 21.346 & 5.032 & 0.032 & 0.640 \\
\hline \multirow[t]{5}{*}{$\mathrm{F}$} & 10 & 37.084 & 8.742 & 1.258 & 12.580 \\
\hline & 10 & 42.234 & 9.956 & 0.044 & 0.440 \\
\hline & 10 & 41.942 & 9.887 & 0.113 & 1.130 \\
\hline & 10 & 42.145 & 9.935 & 0.065 & 0.650 \\
\hline & 10 & 41.895 & 9.876 & 0.124 & 1.240 \\
\hline \multirow[t]{5}{*}{$\mathrm{G}$} & 17 & 72.268 & 17.036 & 0.036 & 0.211 \\
\hline & 17 & 56.271 & 13.265 & 3.735 & 21.971 \\
\hline & 17 & 73.622 & 17.355 & 0.355 & 2.088 \\
\hline & 17 & 73.202 & 17.256 & 0.256 & 1.506 \\
\hline & 17 & 74.826 & 17.639 & 0.639 & 3.759 \\
\hline
\end{tabular}

a cross-correlation method for the location of continuous AE sources. The experiment first establishes an acquisition platform of AE signal based on NI high-speed data acquisition card, and analyzes the acquisition of AE signals of plywood by the way of simulating the sound source with breaking the lead core. Meanwhile, it is decomposed and reconstructed by wavelet analysis, and the weak AE signal which is submerged in noise is extracted and provides an effective signal for researching on the AE source localization. As the measurement and calculation results shown that the number of effective datum in thirty groups of data is up to 27 groups (about 90\%), and the relative error is controlled within $4 \%$. It is proved that the cross-correlation method which is exceedingly suitable for the positioning of $\mathrm{AE}$ source can accurately locate the AE signal of the plywood.

The AE technology can effectively detect the internal defects of plywood for nondestructive testing. Moreover, the wavelet transform offers an optimized way to achieve signal-to-noise separation of AE signal, and provides a true and useful signal datum for AE sources' location. However, although the wavelet transform has multiple wavelet functions to be chosen, its properties are fixed once the wavelet basis function is selected, and the wavelet functions at different scales are obtained by scale and translation transformation. The length of the approximation signals and details will be reduced by half since the signal is broken down once. Meanwhile, there are differences between the approximation signal characteristics obtained at different scales. It is difficult to accurately approximate the local signal characteristics on different scales using the wavelet function derived by a basis function in wavelet transform. Therefore, the reconstructed signal at the time of noise reduction pretreatment will lose some of the original time domain features; on this basis, the method needs to be further improved.

The experiment applies cross-correlation technology only to locate the AE source on the plywood surface, it is possible 
to study the AE signal source location on the surface of fir, pine and other structural timbers, and to explore the characteristics of AE signals with different internal damage defects in the next step. Also combining with the results of this paper, the characteristic parameters of different types of AE signals among Glulam are established and a reference for researching the internal damage and the location of the fracture areas at different materials is provided.

Acknowledgements This work is financially supported by The Natural Science Foundation of China (31100424), The Natural Science Foundation of China (31760182) and Science and Technology Innovation Fund of Southwest Forestry University (C16055).

\section{References}

1. Sanabria SJ (2015) Damage evolution in wood: synchrotron radiation micro-computed tomography $(\mathrm{SR} \mu \mathrm{CT})$ as a complementary tool for interpreting acoustic emission (AE) behavior. Holzforschung 69(3):357-365

2. Verstrynge E, Van Balen K, Wevers M, Ghiassi B, Olierira DV (2016) Detection and localization of debonding damage in composite-masonry strengthening systems with the acoustic emission technique. Nat 483(7391):570-575

3. Chacon J LF, Kappatos V, Balachandran W, Gan TH (2015) A novel approach for incipient defect detection in rolling bearings using acoustic emission technique. Appl Acoustics 89(89):88-100

4. Mostafapour A, Davoodi S (2013) Leakage locating in underground high pressure gas pipe by acoustic emission method. J Nondestruct Evaluat 32(2):113-123

5. Liu K, Lim HB, Frazzoli E, Ji H, Lee VCS (2014) Improving positioning accuracy using GPS pseudorange measurements for cooperative vehicular localization. IEEE Trans Veh Technol 63(6):2544-2556

6. Tsuchikawa S, Kobori H (2015) A review of recent application of near infrared spectroscopy to wood science and technology. J Wood Sci 61(3):213-220

7. Li B, Asikkala J, Filpponen I, Argyropoulos DS (2015) Factors affecting wood dissolution and regeneration of ionic liquids. Ind Eng Chem Res 49(5):2477-2484

8. Pandey KK (1999) A study of chemical structure of soft and hardwood and wood polymers by FTIR spectroscopy. J Appl Polym Sci 71(12):1969-1975

9. Scholz C (1968) Experimental study of the fracturing process in brittle rock. J Geophys Res 73(5):1447-1454

10. Duff AL, Hamdi SE, Plantier G, Lascoup B (2015) Time delay estimation for acoustic source location by means of short-time cross-correlation. Sens IEEE 1885-1888

11. Mostafapour A, Davoodi S, Ghareaghaji M (2014) Acoustic emission source location in plates using wavelet analysis and cross time frequency spectrum. Ultrasonics 54(8):2055-2062
12. Lu S, Jiang M, Sui Q, Dong H, Sai Y, Jia L (2016) Acoustic emission location on aluminum alloy structure by using FBG sensors and PSO method. J Mod Optics 63(8):1-8

13. Zhang P, Zhu J (2014) A novel acoustic emission source localization algorithm based on Gabor wavelet transform and threshold analysis[J]. Piezoelectrics Acoustooptics (4):531-537

14. Huang XH, Zhang YB, Tian BZ, Liu XX (2015) Time delay estimation and acoustic emission source location of rock based on phase difference. Rock Soil Mech (2):381-386

15. Shen KN, Zhao HL, Ding XZ, Ming L, University SF (2015) Acoustic emission signal source localization in wood surface with triangle positioning method. J Northeast For Univ 43(4):77-81

16. Li X, Luo GX, Long SR, Chen G, Li Q (2016) Location method of acoustic emission by time reversal focusing and enhancing for steel plate. Chi J Sci Instrum 37(8):1792-1799

17. Guan JL, Zhao XH, Ren Y, Qi ZH, Lu WW, Zhu SG (2015) Monitoring and analysis of KDP crystal surface waviness. Technol Test (9): 126-131

18. Zhang PL, Li M, Wang RJ, Jiang Y (2016) Acoustic emission research of wood-power blades composites in three point bending test. Eng Plastics Appl 44(4):21-26

19. Shen KN, Zhao HL, Ding XZ, Ming Li (2015) Acoustic emission signal wavelet disjunction in wood damage and fracture process. J Henan Univ Sci Technol: Nat Sci 36(3):33-37

20. Asari D, Hasegawa H, Kanai H (2014) Improvement of myocardial displacement estimation using subkernels for cross correlation between ultrasonic RF echoes. Jpn J Appl Phys 53(7S):07KF21

21. Mao JD, Hundal LS, Thompson ML, Schmidt-Rohr K (2016) Correlation of poly(methylene)-rich amorphous aliphatic domains in humic substances with sorption of a nonpolar organic contaminant phenanthrene. Environ Sci Technol 36(5):929-936

22. Fan BN, Wang HD, Xu BS, Zhang YB (2015) Present research situation of the extraction and processing of weak acoustic emission signals understrong background noise. J Vib Shock 34(16): $147-155$

23. Kulkarni JR (2015) Wavelet analysis of the association between the Southern oscillation and the Indian summer monsoon. Int $\mathbf{J}$ Climatol 20(1):89-104

24. Short AD, Trembanis AC (2015) Decadal scale patterns in beach oscillation and rotation Narrabeen beach, Australia - time series, PCA and wavelet analysis. J Coast Res 20(2):523-532

25. de SobralCintra RJ, Tchervensky RJ, Dimitrov IV, Mintchev VS MP (2016) Wavelet analysis in a canine model of gastric electrical uncoupling. Physiol Meas 25(6):1355-1369

26. Zhou XC, Wang ML, Shi LF, Zhou LF (2015) Diffusion denoising model based on the wavelet and biharmonic equation. Acta Physica Sinica 64(6):064203-1-064203-9

27. Yan R, Shao L, Liu Y (2013) Nonlocal hierarchical dictionary learning using wavelets for image denoising. IEEE Trans Image Process 22(12):4689 